\section{Leiomyoma of the jejunum diagnosed by capsule endoscopy in a pediatric patient}

\begin{abstract}
A 14-year-old girl had been enjoying good health until December 2001, when she received a minor blunt trauma against the left side of the body during gymnastic exercises. Thereafter, she developed intermittent pain in the epigastrium, as well as fatigue, dizziness, and paleness. There was no melena or bright red blood per rectum, but the color of the stool changed to maroon. In June 2002, gastroscopy and colonoscopy revealed no abnormalities. Because of persistent symptoms and iron-deficiency anemia, upper endoscopy was repeated in October 2002, again with no obvious source for the bleeding being found.
\end{abstract}

After another 2 months, she was admitted to hospital for further evaluation of obscure gastrointestinal bleeding. On presentation, the patient reported continuing symptoms. The physical examination did not reveal any abnormalities, apart from pale lips. Laboratory tests showed reduced values for hemoglobin $(8.8 \mathrm{~g} / \mathrm{dl}$; normal range $12-16 \mathrm{~g} / \mathrm{dl})$, mean corpuscular hemoglobin (21 pg; normal range 27-32 pg), mean corpuscular volume ( $70 \mathrm{fl}$; normal range $82-92 \mathrm{fl}$ ), total protein $(5.4 \mathrm{~g} / \mathrm{dl}$; normal range $6.0-8.0 \mathrm{~g} / \mathrm{dl})$, albumin $(3.2 \mathrm{~g} / \mathrm{dl}$; normal range $3.5-$ $5.0 \mathrm{~g} / \mathrm{dl})$ and serum ferritin $(<5 \mathrm{ng} / \mathrm{ml}$; normal range $10-120 \mathrm{ng} / \mathrm{ml}$ ).

A capsule endoscopy (CE) examination was carried out, which identified a large tumor with an irregular surface $11 \mathrm{~min}$ after the pylorus had been passed (Figure 1). A push enteroscopy examination was performed, which confirmed a neoplasia $50-80 \mathrm{~cm}$ after the ligament of Treitz (Figure 2). Biopsies showed that the lesion was an ulcerated mesenchymal neoplasm with leiomyomatous differentiation.

In January 2003, the girl underwent laparoscopic resection of the tumor. Histology showed a completely resected mesenchymal tumor of the jejunal wall, measuring $4 \mathrm{~cm}$ in diameter, with infiltration of the mucosa and superficial ulcera-

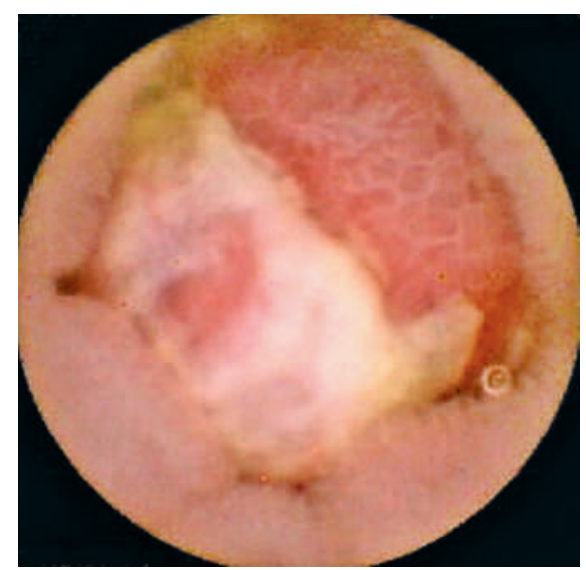

Figure 1 Capsule-endoscopic image of an irregular tumor of the jejunum with an unclear malignant status, with a mucin layer.

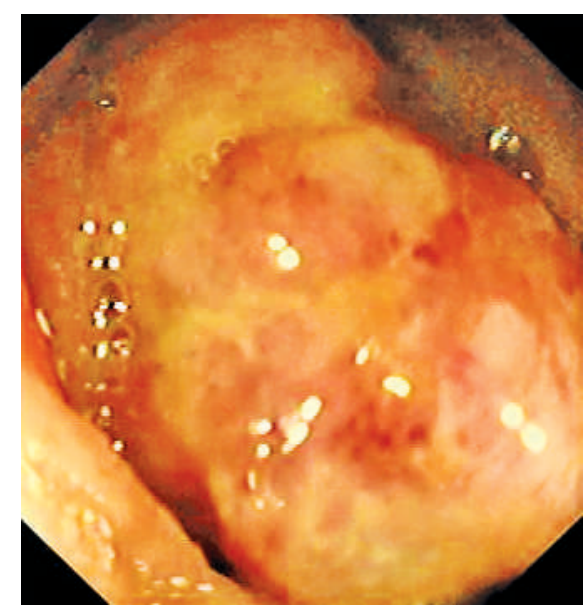

Figure 2 Confirmation of the tumor on push enteroscopy.

tion, but with no vascular invasion (Figure 3a). Immunohistochemistry confirmed leiomyomatous differentiation (Figure $\mathbf{3 b}$ ). The patient was discharged 8 days after surgery in good condition.

Three years after the resection, there have been no signs of recurrence of the tumor and there have been no further symptoms.

Two studies have been published focusing on $C E$ in children [1,2]. Taking these together, CE was safely performed in 42 children over the age of 10 and showed a high diagnostic yield. The main indication
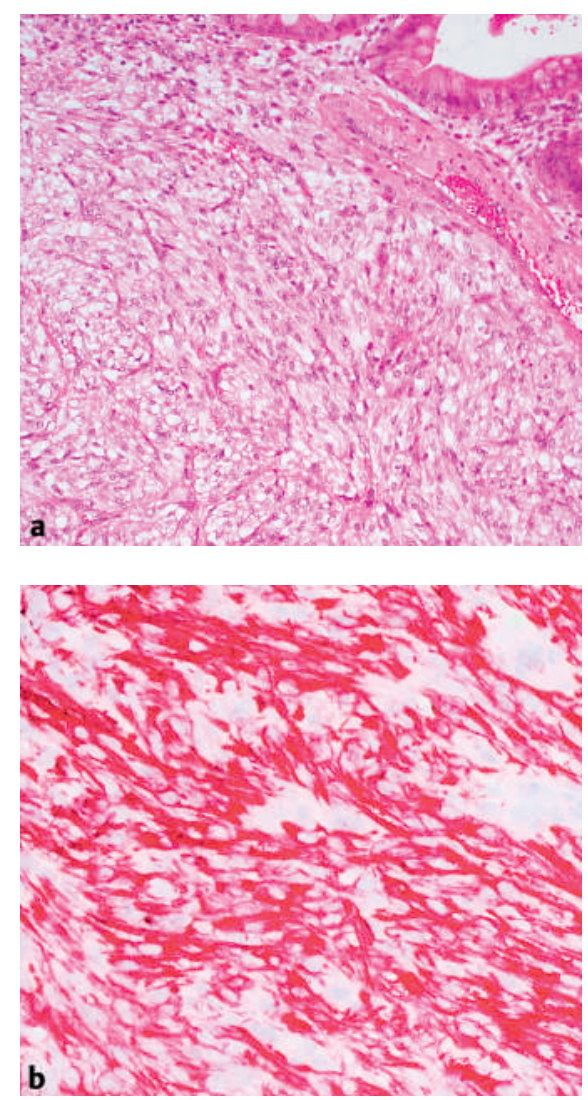

Figure 3 a Histology shows a spindle-cell neoplasm immediately under the intestinal epithelium (hematoxylin-eosin, original magnification $\times 40$ ). b Immunohistochemistry reveals strong reactivity for desmin, confirming leiomyomatous differentiation (immunoalkaline phosphatase, original magnification $\times 200$ ).

for $\mathrm{CE}$ in the two trials was a clinical suspicion of Crohn's disease. In the small group of children who had obscure gastrointestinal bleeding $(\mathrm{n}=4)$, CE confirmed a diagnosis of vascular malformations in three cases [1]. In the present case, CE revealed a tumor of the jejunum with unclear malignant status 1 year after the onset of the initial symptoms. Leiomyomas of the intestine are rarely found in pediatric patients and usually appear in late adult life. To date, there have only been a few reports on smooth-muscle tumors of the small bowel in immunocompetent children [3-6].

DOI: $10.1055 / s-2006-944673$ 


\section{UCTN}

Endoscopy_UCTN_Code_CCL_1AB_2AZ_3AB

Endoscopy_UCTN_Code_CCL_1AC_2AC

S. von Delius ${ }^{1}$, H. Feussner ${ }^{2}$,

E. Frimberger ${ }^{1}$, F. Fend ${ }^{3}$, T. Rösch ${ }^{4}$,

R. M. Schmid ${ }^{1}$, B. Neu ${ }^{1}$

${ }^{1}$ Dept. of Internal Medicine II, Klinikum

rechts der Isar

${ }^{2}$ Dept. of Surgery, Klinikum rechts der Isar

${ }^{3}$ Dept. of Pathology, Klinikum rechts der Isar,

Technical University of Munich, Munich, Germany

${ }^{4}$ Central Interdisciplinary Endoscopy, Virchow Hospital Campus, Charité, Berlin, Germany.
References

${ }^{1}$ Guilhon de Araujo Sant'Anna AM, Dubois J, Miron MC, Seidman EG. Wireless capsule endoscopy for obscure small-bowel disorders: final results of the first pediatric controlled trial. Clin Gastroenterol Hepatol 2005; 3: $264-270$

2 Arguelles-Arias F, Caunedo A, Romero J et al. The value of capsule endoscopy in pediatric patients with a suspicion of Crohn's disease. Endoscopy 2004; 36: 869-873

${ }^{3}$ Ameh EA, Shehu SM, Rafindadi AH, Nmadu PT. Small intestinal leiomyoma in childhood: a case report. West Afr J Med 2002; 21: $157-158$

${ }^{4}$ Tervit GJ, Forster AL. Leiomyoma of the small intestine in an 11-year-old boy. Eur J Pediatr Surg 1997; 7: 44

${ }^{5}$ Chu MH, Lee HC, Shen EY et al. Gastrointestinal bleeding caused by leiomyoma of the small intestine in a child with neurofibromatosis. Eur J Pediatr 1999; 158: 460-462

${ }^{6}$ Freeman J. Leiomyoma of small bowel: a case report. J Pediatr Surg 1979; 14: 477 478
Corresponding author

\section{S. von Delius, M.D.}

II. Medizinische Klinik Klinikum Rechts der Isar Ismaninger Straße 22 81675 München

Germany

E-mail: stefan_ruckert@yahoo.de 